\title{
Covid-19 e Câncer: Atualização de Aspectos Epidemiológicos
}

doi: https://doi.org/10.32635/2176-9745.RBC.2020v66nTemaAtual.1013

\author{
COVID-19 and Cancer: Updating Epidemiological lssues \\ Covid-19 y Cáncer: Actualización de Aspectos Epidemiológicos
}

\begin{abstract}
Jeniffer Dantas Ferreira'; Fernanda Cristina da Silva de Lima²; Julio Fernando Pinto Oliveira³; Marianna de Camargo Cancela; Marceli de Oliveira Santos ${ }^{5}$
\end{abstract}

Resumo

Introduçáo: A doença causada pelo novo coronavírus (Covid-19) é atualmente um problema mundial de saúde pública. A idade avançada, sexo masculino, histórico de tabagismo e presença de comorbidades, entre as quais, o câncer, foram relatados na literatura como fatores associados ao pior prognóstico da doença. Objetivos: Revisar a literatura acerca da infecção pelo novo coronavírus em indivíduos portadores de neoplasias malignas e atualizar os aspectos epidemiológicos da doença em pacientes oncológicos. Método: Revisão de trabalhos publicados em periódicos científicos que abordavam os aspectos epidemiológicos da infecçáo por coronavírus em pacientes oncológicos por meio de termos do Medical Subject Headings (MeSH) e dos Descritores em Ciências da Saúde (DeCs) na base de dados MEDLINE/ PubMed. Resultados: Pacientes com câncer apresentam piores resultados clínicos quando comparados à população geral. Conclusáo: Pacientes oncológicos idosos, portadores de câncer de pulmão, ou que se submeteram à cirurgia oncológica ou à quimioterapia recentes ao diagnóstico de Covid-19 apresentaram maior suscetibilidade ao desenvolvimento da infecção grave.

Palavras-chave: Neoplasias; Vírus da SARS; Infecçôes por Coronavírus; Epidemiologia; Pandemias.

\section{Abstract}

Introduction: The disease caused by the new coronavirus (COVID-19) is currently a global public health issue. Advanced age, male gender, history of tobacco addiction and presence of comorbidities, among them cancer, were reported in the literature as factors associated to the worse prognosis of the disease. Objectives: To review the literature about the new coronavirus infection in individuals with malignant neoplasms and to update the epidemiological aspects of the disease in oncologic patients. Method: Literature review on articles published in scientific journals that addressed the epidemiologic aspects of the infection by coronavirus in oncologic patients using the terms of the Medical Subject Headings (MeSH) and of the Health Sciences Descriptors (DeCs) in the MEDLINE/PubMed database. Results: Patients with cancer have worse clinical results when compared to the general population. Conclusion: Elderly oncologic patients with lung cancer or patients who have submitted to recent cancer surgery or chemotherapy when diagnosed with COVID-19 were more susceptible to the development of severe infection.

Key words: Neoplasms; SARS Virus; Coronavirus Infections; Epidemiology; Pandemics.

\section{Resumen}

Introducción: La enfermedad causada por coronavirus (Covid-19) es actualmente un problema de salud pública. Las personas con enfermedades crónicas como el cáncer, el sexo masculino, los pacientes con antecedentes de tabaquismo y los ancianos tienen un mayor riesgo de adquirir formas graves, desarrollar complicaciones y morir por la enfermedad. Objetivos: Revisar la literatura sobre la infección con el nuevo coronavirus en individuos con neoplasias malignas y sintetizar los aspectos epidemiológicos de la enfermedad en pacientes con cáncer. Método: Revisión de la literatura como artículos publicados en revistas científicas utilizando los términos médicos (MeSH) y Descriptores en Ciencia de la Salud (DeCs) en la base de datos MEDLINE/PubMed. Resultados: Los pacientes con cáncer tienen peores resultados clínicos en comparación con la población general. Conclusiones: Pacientes oncológicos ancianos, portadores de cáncer de pulmón, o que se sometieron a cirugía de cáncer o a quimioterapia reciente al diagnóstico de Covid-19 fueron más susceptibles al desarrollo de infección severa. Palavras clave: Neoplasias; Virus del SRAS; Infecciones por Coronavirus; Epidemiología; Pandemias.

\footnotetext{
${ }^{1}$ Doutora. Enfermeira. Divisão de Vigilância e Análise de Situação da Coordenação de Prevenção e Vigilância (Conprev) do Instituto Nacional de Câncer José Alencar Gomes da Silva (INCA). Rio de Janeiro (RJ), Brasil. Orcid iD: https://orcid.org/0000-0002-6724-5614

${ }^{2}$ Mestre. Estatística. Divisão de Vigilância e Análise de Situação. Conprev/INCA. Rio de Janeiro (RJ), Brasil. Orcid iD: https://orcid.org/0000-0002-7815-4304

${ }^{3}$ Mestre. Estatístico. Divisão de Vigilância e Análise de Situação. Conprev/INCA. Rio de Janeiro (RJ), Brasil. Orcid iD: https://orcid.org/0000-0002-9187-527X

${ }^{4}$ Doutora. Dentista. Divisão de Vigilância e Análise de Situação. Conprev/INCA. Rio de Janeiro (RJ), Brasil. Orcid iD: https://orcid.org/0000-0002-8169-8054

${ }^{5}$ Doutora. Estatística. Divisão de Vigilância e Análise de Situação. Conprev/INCA. Rio de Janeiro (RJ), Brasil. Orcid iD: https://orcid.org/0000-0001-5197-2019

Endereço para correspondência: Marceli de Oliveira Santos. Rua Marquês de Pombal 125 - $6^{\circ}$ andar - Centro. Rio de Janeiro (RJ), Brasil. CEP $20230-240$.

E-mail: msantos@inca.gov.br
} 


\section{INTRODUÇÃO}

Em dezembro de 2019, foram identificados os primeiros casos da doença pelo coronavírus 2019 (coronavirus disease 2019 - Covid-19), ocasionada pela presença de um novo coronavírus, da síndrome respiratória aguda grave do coronavírus 2 (severe acute respiratory syndrome coronavirus 2 -Sars-CoV-2), originário de Wuhan, Hubei, na China ${ }^{1}$. A idade avançada, sexo masculino, histórico de tabagismo e presença de comorbidades, entre as quais, o câncer, foram relatados na literatura como os fatores associados ao pior prognóstico da doençą ${ }^{2}$. A rápida progressão da pandemia de Covid-19 foi declarada pela Organizaçáo Mundial da Saúde (OMS) como uma Emergência de Saúde Pública de Importância Internacional ${ }^{3}$. Por ser uma doença com alto potencial de transmissibilidade, por meio de gotículas ao tossir, espirrar ou falar, condutas de distanciamento social, quarentena e isolamento foram adotados em diversos países, além de instruções de higiene e etiqueta respiratória ${ }^{3-4}$.

Os sintomas mais frequentes são febre (acima de $37,8^{\circ} \mathrm{C}$ ), dificuldade e/ou desconforto respiratório, tosse seca, dores de cabeça, diarreia, dores musculares, anosmia e ageusia. Embora cerca de $80 \%$ dos infectados sejam assintomáticos ${ }^{3}$, imunossuprimidos, idosos e portadores de doenças crônicas, entre as quais, o câncer, estão mais susceptíveis a complicaçôes graves da síndrome respiratória aguda, com evolução para a síndrome da disfunção múltipla de órgãos, que inclui insuficiência respiratória e renal ${ }^{5-8}$.

Entre os pacientes com câncer, os que apresentaram maior risco de complicaçóes em razão da infecção por coronavírus foram os portadores de câncer de pulmão, os que passaram por transplante de medula óssea ou que fizeram tratamento quimioterápico ${ }^{5,9,10}$. Portanto, a pergunta de pesquisa que norteou o estudo foi: Qual perfil epidemiológico dos pacientes oncológicos que desenvolveram Covid-19? O objetivo foi compilar as informaçóes de publicaçôes sobre a infecção por Sars-CoV-2 em pacientes oncológicos com o intuito de alertar e direcionar a assistência prestada por familiares, cuidadores e profissionais de saúde.

\section{MÉTODO}

Para atender aos objetivos propostos, optou-se por um estudo de revisão de literatura. Foram selecionados os trabalhos publicados em periódicos científicos que abordavam os aspectos epidemiológicos da infecção por coronavírus em pacientes oncológicos. Os critérios de seleção foram os documentos em idiomas inglês, português, espanhol e francês; disponíveis na íntegra; publicados entre $1^{\circ}$ de dezembro de 2019 e 22 de abril de 2020; encontrados na base de dados MEDLINE/ PubMed, utilizando os descritores controlados do Medical Subject Headings (MeSH) e dos Descritores em Ciências da Saúde (DeCS), a partir do operador booleano AND, bem como seus respectivos sinônimos por meio do operador booleano OR.

A combinação de termos Covid-19 e câncer suscitou em 198 publicaçôes ((Severe Acute Respiratory Syndrome Coronavirus 2[Supplementary Concept] OR COVID-19[Supplementary Concept] OR Severe Acute Respiratory Syndrome Coronavirus 2[tiab] OR Coronavirus Disease 2019[tiab] OR 2019 Novel Coronavirus[tiab] OR Wuhan Coronavirus[tiab] OR COVID-19[tiab] OR SARS-CoV-2[tiab] OR 2019-nCoV[tiab]) AND (Neoplasms[mh] OR Neoplas*[tiab] OR Cancer*[tiab] OR Tumor*[tiab] OR Tumour*[tiab] OR Carcinoma[mh] OR Carcinoma*[tiab] OR Malignan*[tiab] OR Medical Oncology[mh] OR Oncol*[tiab])) AND (English[lang] OR Portuguese[lang] OR Spanish[lang] OR French[lang]).

Considerou-se critério de exclusão aqueles sem relação com objeto de estudo após a leitura do resumo e os que não abordassem a população-alvo do estudo, pacientes oncológicos. Procedeu-se à busca em literatura cinzenta incluindo relatórios governamentais, bem como foram utilizadas outras referências para elaboração da introdução e discussão dos resultados.

\section{RESULTADOS}

Os títulos e resumos dos artigos foram avaliados por quatro revisores, obedecendo aos critérios de exclusão e inclusão preestabelecidos, a fim de evitar vieses na sua seleção. Os casos discordantes $(\mathrm{N}=2)$ foram deliberados por consenso, resultando na seleção final de 13 artigos para a leitura na íntegra e elaboraçáo deste estudo. As informações coletadas estão apresentadas no Quadro 1.

Em sua totalidade, os artigos foram publicados em 2020. No que se refere ao tipo de publicação, $69,2 \%$ $(\mathrm{N}=9)$ eram artigos originais, $15,4,0 \%(\mathrm{~N}=2)$ cartas ao editor e $15,4 \%(\mathrm{~N}=2)$ editoriais. Entre os delineamentos de estudos realizados, 30,7\% $(\mathrm{N}=4)$ foram estudos transversais, $23,1 \%(\mathrm{~N}=3)$ estudos de coorte, $23,1 \%$ $(\mathrm{N}=3)$ revisóes sistemáticas com metanálises, $15,4 \%$ $(\mathrm{N}=2)$ opinião de experts e $7,7 \%(\mathrm{~N}=1)$ estudo de caso, cujos níveis de evidência $2 \mathrm{a}, 2 \mathrm{~b}, 4$ e 5 foram identificados em $23,1 \%(\mathrm{~N}=3), 30,7 \%(\mathrm{~N}=4), 7,7 \%(\mathrm{~N}=1)$ e $38,5 \%$ $(\mathrm{N}=5)$, respectivamente ${ }^{11}$.

O cenário de estudo mais frequente foi a China $(\mathrm{N}=9$; $69,2 \%)$, tendo sido identificadas outras localidades como 
Quadro 1. Descrição das publicações selecionadas segundo autoria, tipo de publicação, tipo de câncer, título, cenário, desenho e população de estudo e medidas de efeito

\begin{tabular}{|c|c|c|c|c|c|c|c|}
\hline $\mathbf{N}^{\circ}$ & Autor/ano & Título & $\begin{array}{c}\text { Tipo de } \\
\text { publicação }\end{array}$ & $\begin{array}{l}\text { Cenário, desenho } \\
\text { e população de } \\
\text { estudo }\end{array}$ & $\begin{array}{l}\text { Nível de } \\
\text { evidência }^{a}\end{array}$ & $\begin{array}{l}\text { Tipo de } \\
\text { câncer }\end{array}$ & Medidas de efeito \\
\hline 1 & $\begin{array}{l}\text { Liang et al., } \\
2020\end{array}$ & $\begin{array}{l}\text { Cancer patients in } \\
\text { SARS-CoV-2 infection: } \\
\text { a nationwide analysis } \\
\text { in China }\end{array}$ & Artigo & $\begin{array}{l}\text { China, estudo de } \\
\text { coorte prospectivo } \\
\text { multicêntrico, } \\
\mathrm{N}=1.590\end{array}$ & $2 b$ & $\begin{array}{l}\text { Qualquer } \\
\text { neoplasia } \\
\text { maligna }\end{array}$ & $\begin{array}{l}\text { Cuidados intensivos: } \\
\mathrm{P}=39 \%(\mathrm{~N}=18) \text { dos } \\
\text { pacientes com câncer } \\
\text { versus } 8 \%(\mathrm{~N}=1572) \\
\text { dos pacientes sem câncer } \\
\quad(\mathrm{p}=0,0003)\end{array}$ \\
\hline 2 & Xia et al., 2020 & $\begin{array}{l}\text { Risk of COVID-19 for } \\
\text { patients with cancer }\end{array}$ & Carta ao editor & $\begin{array}{c}\text { China, estudo } \\
\text { transversal, } \mathrm{N}=1.590\end{array}$ & 5 & $\begin{array}{l}\text { Qualquer } \\
\text { neoplasia } \\
\text { maligna }\end{array}$ & - \\
\hline 3 & Yu et al., 2020 & $\begin{array}{l}\text { SARS-CoV-2 transmission } \\
\text { in patients with cancer at } \\
\text { a tertiary care hospital in } \\
\text { Wuhan, China }\end{array}$ & Artigo & $\begin{array}{l}\text { China, estudo } \\
\text { transversal, } \mathrm{N}=1.524\end{array}$ & $2 b$ & $\begin{array}{l}\text { Qualquer } \\
\text { neoplasia } \\
\text { maligna }\end{array}$ & $\begin{array}{c}\text { Taxa de infecção por } \\
\text { coronavírus em pacientes } \\
\text { oncológicos: 0,79\% } \\
\text { (IC95\% 0,3\%-1,2\%) }\end{array}$ \\
\hline 4 & $\begin{array}{c}\text { Shankar et al., } \\
2020\end{array}$ & $\begin{array}{l}\text { Cancer care delivery } \\
\text { challenges amidst } \\
\text { coronavirus disease - } 19 \\
\text { (COVID-19) outbreak: } \\
\text { specific precautions for } \\
\text { cancer patients and } \\
\text { cancer care providers to } \\
\text { prevent spread }\end{array}$ & Editorial & $\begin{array}{l}\text { Índia, opinião de } \\
\text { experts }\end{array}$ & 5 & $\begin{array}{l}\text { Qualquer } \\
\text { neoplasia } \\
\text { maligna }\end{array}$ & - \\
\hline 5 & $\begin{array}{l}\text { Guan et al., } \\
2020\end{array}$ & $\begin{array}{l}\text { Comorbidity and its } \\
\text { impact on } 1590 \text { patients } \\
\text { with covid-19 in China: a } \\
\text { nationwide analysis }\end{array}$ & Artigo & $\begin{array}{l}\text { China, estudo de } \\
\text { caso retrospectivo } \\
\text { multicêntrico, } \\
\mathrm{N}=1.524\end{array}$ & 4 & $\begin{array}{l}\text { Qualquer } \\
\text { neoplasia } \\
\text { maligna }\end{array}$ & $\begin{array}{c}\mathrm{HR}=1,79(\mathrm{IC} 95 \% \\
1,16-2,77)\end{array}$ \\
\hline 6 & $\begin{array}{l}\text { Zhang et al., } \\
2020\end{array}$ & $\begin{array}{l}\text { Clinical characteristics } \\
\text { of COVID-19-infected } \\
\text { cancer patients: a } \\
\text { retrospective case study } \\
\text { in three hospitals within } \\
\text { Wuhan, China }\end{array}$ & Artigo & $\begin{array}{l}\text { China, estudo de } \\
\text { coorte retrospectivo } \\
\text { multicêntrico, N=28 }\end{array}$ & $2 b$ & $\begin{array}{c}\text { Pulmão, } \\
\text { esôfago, } \\
\text { mama, } \\
\text { laringe, fígado, } \\
\text { próstata, colo } \\
\text { do útero, } \\
\text { cólon, reto, } \\
\text { nasofaringe, } \\
\text { endométrio, } \\
\text { ovário e } \\
\text { testículo }\end{array}$ & $\begin{array}{l}\text { Tipos de câncer mais } \\
\text { frequentes: pulmão } \\
(25,0 \%) \text {, esôfago } \\
(14,3 \%) \text { e mama } \\
(10,7 \%) \text {. Terapia } \\
\text { antitumoral até } 14 \text { dias } \\
\text { antes da infecção por } \\
\text { Covid-19: } 21,4 \%\end{array}$ \\
\hline 7 & Shi et al., 2020 & $\begin{array}{l}\text { Host susceptibility to } \\
\text { severe COVID-19 and } \\
\text { establishment of a host } \\
\text { risk score: findings of } \\
487 \text { cases outside Wuhan }\end{array}$ & Artigo & $\begin{array}{l}\text { China, estudo de } \\
\text { coorte retrospectivo, } \\
\qquad N=487\end{array}$ & $2 b$ & $\begin{array}{l}\text { Qualquer } \\
\text { neoplasia } \\
\text { maligna }\end{array}$ & $\begin{array}{c}\text { Casos severos de } \\
\text { Covid-19: } P=4,1 \% \\
\text { pacientes oncológicos } \\
\text { versus } 0,7 \% \text { pacientes } \\
\text { sem comorbidades } \\
\quad(p=0,025)\end{array}$ \\
\hline 8 & $\begin{array}{l}\text { Emami et al., } \\
2020\end{array}$ & $\begin{array}{l}\text { Prevalence of underlying } \\
\text { diseases in hospitalized } \\
\text { patients with covid-19: } \\
\text { a systematic review and } \\
\text { meta-analysis }\end{array}$ & Artigo & $\begin{array}{l}\text { Irã, revisão } \\
\text { sistemática e } \\
\text { metanálise, } \\
\mathrm{N}=76.993\end{array}$ & $2 a$ & $\begin{array}{l}\text { Qualquer } \\
\text { neoplasia } \\
\text { maligna }\end{array}$ & $\begin{array}{c}\mathrm{P}=0,92(\mathrm{IC} 95 \% 0,56- \\
1,34)\end{array}$ \\
\hline 9 & $\begin{array}{l}\text { Wang et al., } \\
2020\end{array}$ & $\begin{array}{l}\text { Does comorbidity } \\
\text { increase the risk of } \\
\text { patients with COVID-19: } \\
\text { evidence from meta- } \\
\text { analysis }\end{array}$ & Artigo & $\begin{array}{c}\text { China, revisão } \\
\text { sistemática e } \\
\text { metanálise, } \mathrm{N}=1.558\end{array}$ & $2 a$ & $\begin{array}{l}\text { Qualquer } \\
\text { neoplasia } \\
\text { maligna }\end{array}$ & $\begin{array}{c}\mathrm{OR}=2,29(\mathrm{IC} 95 \%: 1,00- \\
5,23, \mathrm{p}=0,627)\end{array}$ \\
\hline
\end{tabular}




\begin{tabular}{|c|c|c|c|c|c|c|c|}
\hline $\mathbf{N}^{\circ}$ & Autor/ano & Título & $\begin{array}{c}\text { Tipo de } \\
\text { publicação }\end{array}$ & $\begin{array}{c}\text { Cenário, desenho } \\
\text { e população de } \\
\text { estudo }\end{array}$ & $\begin{array}{l}\text { Nível de } \\
\text { evidência }^{a}\end{array}$ & $\begin{array}{l}\text { Tipo de } \\
\text { câncer }\end{array}$ & Medidas de efeito \\
\hline 10 & $\begin{array}{l}\text { Desai et al., } \\
2020\end{array}$ & $\begin{array}{l}\text { COVID-19 and cancer: } \\
\text { lessons from a pooled } \\
\text { meta-analysis }\end{array}$ & Artigo & $\begin{array}{l}\text { Estados Unidos, } \\
\text { revisão sistemática e } \\
\text { metanálise, } \mathrm{N}=3.661\end{array}$ & $2 a$ & $\begin{array}{l}\text { Qualquer } \\
\text { neoplasia } \\
\text { maligna }\end{array}$ & $\begin{array}{c}P=2,0 \%(I C 95 \% 2,0 \%- \\
3,0 \%)\end{array}$ \\
\hline 11 & Ma et al., 2020 & $\begin{array}{l}\text { Clinical characteristics } \\
\text { and prognosis in cancer } \\
\text { patients with COVID-19: } \\
\text { a single center's } \\
\text { retrospective study }\end{array}$ & Carta do editor & $\begin{array}{c}\text { China, estudo } \\
\text { transversal, } \mathrm{N}=1.380\end{array}$ & 5 & $\begin{array}{l}\text { Colorretal, } \\
\text { pulmão, } \\
\text { mama, } \\
\text { ginecológicos } \\
\text { e outras } \\
\text { neoplasias }\end{array}$ & $\begin{array}{c}\mathrm{P}=54,1 \% \text { pacientes } \\
\text { severos de Covid-19 } \\
\text { portadores de câncer. } \\
\text { Comorbidades associadas } \\
\text { e pacientes com eventos } \\
\text { severos } \mathrm{P}=40 \%\end{array}$ \\
\hline 12 & $\begin{array}{l}\text { Novel } \\
\text { Coronavirus } \\
\text { Pneumonia } \\
\text { Emergency } \\
\text { Response } \\
\text { Epidemiology } \\
\text { Team, 2020 }\end{array}$ & $\begin{array}{l}\text { The epidemiological } \\
\text { characteristics of an } \\
\text { outbreak of } 2019 \text { novel } \\
\text { coronavirus diseases } \\
\text { (COVID-19) in China }\end{array}$ & Artigo & $\begin{array}{l}\text { China, estudo } \\
\text { transversal, } \\
\mathrm{N}=72.314\end{array}$ & 5 & $\begin{array}{l}\text { Qualquer } \\
\text { neoplasia } \\
\text { maligna }\end{array}$ & $\begin{array}{l}\text { Taxa de fatalidade em } \\
\text { pacientes oncológicos } \\
5,6 \% \text { versus } 0,9 \% \\
\text { pacientes sem } \\
\text { comorbidades }\end{array}$ \\
\hline 13 & $\begin{array}{l}\text { Thuler e Melo, } \\
2020\end{array}$ & $\begin{array}{l}\text { Sars-CoV-2/Covid-19 em } \\
\text { pacientes com câncer }\end{array}$ & Editorial & $\begin{array}{c}\text { Brasil, opinião de } \\
\text { experts }\end{array}$ & 5 & $\begin{array}{c}\text { Qualquer } \\
\text { neoplasia } \\
\text { maligna }\end{array}$ & - \\
\hline
\end{tabular}

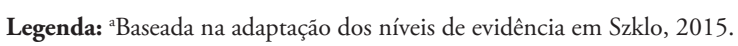

Índia $(\mathrm{N}=1 ; 7,7 \%)$, Irã $(\mathrm{N}=1 ; 7,7 \%)$, Estados Unidos $(\mathrm{N}=1 ; 7,7 \%)$ e Brasil $(\mathrm{N}=1 ; 7,7 \%)$.

Em relação à gravidade da doença Covid-19, foram observados casos mais severos em pacientes oncológicos. Essa avaliação foi realizada e apresentada nos artigos 1, 3, 7, 11 e 12 relacionados no Quadro 1.

\section{DISCUSSÃO}

Das publicaçôes selecionadas, cinco revelaram maior prevalência de portadores de câncer em casos confirmados de Covid-19 comparados à população geral $(1,0 \%$ versus $0,29 \%)^{5}$. Yu et al. ${ }^{12}$ referiram chance duas vezes maior na suscetibilidade da infecção por pacientes com câncer $\left(\mathrm{OR}=2,31\right.$; IC95\% 1,89-3,02). Shankar et al. ${ }^{2}$ sugerem que a magnitude do risco de infecção por Covid-19 seja duas vezes maior para portadores de neoplasia maligna frente à população geral. Em metanálise, Desai et al. ${ }^{13}$ também referem prevalência duas vezes maior em pacientes com câncer infectados por Covid-19 ( $\mathrm{P}=2,0 \%$; IC95\% 2,0\%-3,0\%). Em revisão, Thuler e Melo ${ }^{14}$ sugerem que os indivíduos com câncer apresentam risco mais elevado para o desenvolvimento da doença por Covid-19 comparando-os com a população geral. Em contrapartida, os achados da metanálise conduzida por Wang et al. ${ }^{15}$ não corroboram a correlação entre portadores de neoplasias e a evolução para quadro clínico severo de Covid-19 quando comparados a todos os pacientes incluídos no estudo (OR=2,29; IC95\% 1,00-5,23) ainda que limítrofe.
Os pacientes oncológicos, especialmente aqueles que estão em tratamento, são mais suscetíveis à infecção, tendo em vista que algumas modalidades terapêuticas comprometem seu sistema imunológico ${ }^{16,17}$, sendo corroborado por quatro publicaçóes encontradas nesta revisão. Liang et al. ${ }^{5}$ observaram que $25 \%$ dos 16 portadores de câncer haviam sido submetidos ao tratamento em menos de um mês da infecção por Covid-19 e, em $75 \%$ de 18 pacientes, foi realizada ressecção primária do tumor no mês anterior à infecção. Foi observado por Zhang et al. ${ }^{18}$ que $35,7 \%$ dos pacientes oncológicos $(\mathrm{N}=10)$ apresentaram estadiamento clínico do tumor IV e $21,4 \%(N=6)$ haviam realizado terapia antitumoral em até 14 dias que antecederam o diagnóstico de Covid-19. Ainda neste estudo, foi observado aumento do risco para o desenvolvimento de infecção mais severa em pacientes submetidos à terapia antitumoral $(\mathrm{HR}=4,079$; IC95\% 1,086-15,322, $\mathrm{p}=0,037)$. Dos 12 pacientes oncológicos infectados por Sars-CoV-2 (N=1.254) observados no estudo realizado por $\mathrm{Yu}$ et al. ${ }^{12}, 41,7 \%(\mathrm{~N}=5)$ estavam realizando tratamento (imunoterapia, quimioterapia ou radioterapia). Ma et al. ${ }^{19}$ identificaram que 35\% dos portadores de câncer com infecção por coronavírus moderada ou severa haviam realizado terapia antitumoral em até um mês ao diagnóstico de Covid-19.

Liang et al. ${ }^{5}$ apontaram que 39\% dos pacientes com câncer $(\mathrm{N}=18)$ necessitaram de cuidados intensivos frente a $8 \%(\mathrm{~N}=124)$ dos 1.572 pacientes $(\mathrm{p}=0,0003)$. No estudo conduzido por Shi et al. ${ }^{20}$, dos 487 casos de Covid-19, 49 
$(10,1 \%)$ evoluíram para sintomas severos, no qual 4,1\% eram portadores de neoplasias em comparação a 438 $(89,9 \%)$ que apresentaram sintomas moderados, e $0,7 \%$ eram pacientes oncológicos $(\mathrm{p}=0,025)$. Para Ma et al. ${ }^{19}$, a proporção de casos de Covid-19 severos ou em estado crítico foi de $54,1 \%$ em pacientes com câncer, percentual mais elevado do que o encontrado em estudo feito por Wang et al. ${ }^{21}$ com 138 pacientes com Covid-19, no qual $26,1 \%(\mathrm{~N}=36)$ eram pacientes graves. A taxa de letalidade por coronavírus em pacientes com qualquer neoplasia foi de 5,6\% em estudo conduzido pelo grupo epidemiológico chinês, Novel Coronavirus Pneumonia Emergency Response Epidemiology Team ${ }^{22}$. O estudo realizado por Yu et al. ${ }^{12}$ náo encontrou aumento no risco de infecçóes graves por Covid-19 para pacientes oncológicos.

O câncer de pulmão foi referido por quatro publicações como um dos tipos de câncer possivelmente mais suscetível à infecção por Covid-19. Quando comparados aos 18 pacientes com câncer $(\mathrm{N}=1.590), 28 \%(\mathrm{~N}=5)$ eram portadores de câncer de pulmão, de acordo com Liang et al. ${ }^{5}$. No entanto, náo foi encontrada maior probabilidade de desenvolver infecção grave em pacientes com câncer de pulmão quando comparados a portadores de outras neoplasias - um (20\%) de cinco pacientes com câncer de pulmão versus oito (62\%) de 13 pacientes com outros tipos de câncer $(p=0,294)^{5}$. No estudo conduzido por Zhang et al. ${ }^{18}$, entre os 1.276 pacientes infectados, 28 $(2,2 \%)$ eram portadores de neoplasias malignas, sendo o tipo de câncer mais frequente o de pulmão (em 25\% dos casos), seguido pelo câncer de esôfago ( $\mathrm{N}=4 ; 14,3 \%)$ e mama $(\mathrm{N}=3 ; 10,7 \%)$. Yu et al. ${ }^{12}$ identificaram em sua populaçáo de estudo $58,3 \%$ de pacientes com câncer de pulmão $(\mathrm{N}=12)$. Para Ma et al. ${ }^{19}$, dos 37 indivíduos com câncer e Covid-19, 20 evoluíram para formas graves da infecção. Destes, 35,0\% (N=7) eram portadores de câncer colorretal e 20,0\% ( $\mathrm{N}=4)$ eram pacientes com câncer de pulmão $(\mathrm{N}=8)$. Outros tipos de câncer também foram investigados, mas as diferenças encontradas não foram estatisticamente significativas. Emami et al. ${ }^{16}$, em estudo de revisão sistemática e metanálise $(\mathrm{N}=3.403)$, sugerem que o câncer está entre as doenças mais prevalentes $(0,92 \%$ IC95\% 0,56-1,34).

A análise de pacientes com câncer também portadores de outras comorbidades foi realizada em cinco publicações. No estudo conduzido por Guan et al. ${ }^{23}$, foi utilizada uma medida composta para avaliar a necessidade de cuidados intensivos (CTI e ventilação mecânica) e óbito como medida de risco de gravidade da infecção por Covid-19, e identificou-se risco $79 \%$ maior em indivíduos com qualquer comorbidade, incluindo câncer, diabetes, hipertensão, doenças cardiovasculares e cerebrovasculares quando comparados a indivíduos sem comorbidades $(\mathrm{HR}=1,79$;
IC95\% 1,16-2,77). Para pacientes com duas ou mais comorbidades associadas, o risco foi 2,5 vezes mais elevado (HR=2,59; IC95\% 1,61-4,17). A análise ajustada por idade e tabagismo evidenciou o incremento no risco em 3,5 vezes $\left(\mathrm{HR}=3,5 ;\right.$ IC95\% 1,60-7,64). E, em Zhang et al. ${ }^{18}, 39,2 \%$ $(\mathrm{N}=11)$ apresentaram outras comorbidades associadas ao câncer. Ma et al. ${ }^{19}$ observaram que, entre os portadores de neoplasias malignas e Covid-19 com complicaçóes moderadas ou severas, em 40,0\% ( $\mathrm{N}=8)$ dos casos graves $(\mathrm{N}=20)$, apresentaram mais de uma comorbidade e evoluíram para formas severas da infecção por coronavírus.

Em dois artigos, considerou-se que a evidência de câncer como um fator de risco para desenvolvimento da Covid-19 foi baseada em amostras pequenas, sendo esse fato uma limitação para os achados ${ }^{23,24}$.

Cabe ressaltar o desconhecimento dos efeitos do coronavírus em pacientes oncológicos, no que diz respeito à sobrevida ou à mortalidade em face à descontinuidade ou ao atraso no tratamento, ocasionados pela saturação dos serviços de saúde. Embora não haja ainda evidência científica para alteraçóes no protocolo terapêutico durante a pandemia ${ }^{2,17}$. Mourey et al. ${ }^{25}$ enfatizaram a necessidade de se limitar as internaçóes hospitalares e aplicação de medidas restritivas prévias ao início ou à continuidade do tratamento oncológico em virtude do risco cumulativo pela idade avançada e diagnóstico de câncer.

Uma vez que idade avançada e a presença de comorbidades são comuns aos pacientes oncológicos ${ }^{14}$, as estratégias de cuidado direcionadas a essa parcela da população devem levar em consideração a suscetibilidade ao desenvolvimento da forma mais severa da doença, incluindo medidas de prevenção, detecção precoce, cura e reabilitação da infecção para o planejamento da assistência oncológica $^{16,26,27}$.

\section{CONCLUSÃO}

A análise dos achados sobre o perfil epidemiológico dos pacientes oncológicos que contraíram a Covid-19 foi sintetizada neste estudo. Uma vez que a idade avançada e as comorbidades foram apontadas como fatores de risco, e o câncer pode ser considerado uma doença relacionada ao envelhecimento, pacientes oncológicos são efetivamente mais vulneráveis a desenvolver a Covid-19 em sua foram mais severa.

\section{CONTRIBUIÇÕES}

Todos os autores contribuíram igualmente na concepção, análise e interpretação da pesquisa; na redação e revisão crítica com contribuição intelectual; e na aprovação da versão final. 


\section{DECLARAÇÃO DE CONFLITO DE INTERESSES}

Nada a declarar.

\section{FONTES DE FINANCIAMENTO}

Fernanda Cristina da Silva de Lima, Jeniffer Dantas Ferreira e Julio Fernando Pinto Oliveira são bolsistas institucionais do INCA.

\section{REFERÊNCIAS}

1. Chen N, Zhou M, Dong X, et al. Epidemiological and clinical characteristics of 99 cases of 2019 novel coronavirus pneumonia in Wuhan, China: a descriptive study. Lancet. 2020;395(10223):507-13. doi: https:// doi.org/10.1016/S0140-6736(20)30211-7

2. Shankar A, Saini D, Roy S, et al. Cancer care delivery challenges amidst coronavirus disease - 19 (COVID-19) outbreak: specific precautions for cancer patients and cancer care providers to prevent spread. Asian Pac J Cancer Prev. 2020;21(3):569-573. doi: https://doi. org/10.31557/APJCP.2020.21.3.569

3. Organização Pan-Americana da Saúde [Internet]. Brasília, DF: OPAS; [2000]. Folha informativa - COVID-19 (doença causada pelo novo coronavírus) [acesso 2020 mar 25]. Disponível em: https://www.paho.org/bra/ index.php?option $=$ com_content $\&$ view $=$ article\&id $=61$ 01: covid19\&Itemid $=875$

4. Ministério da Saúde [Internet]. Brasília, DF: Ministério da Saúde; [2020]. O que é coronavírus? [acesso 2020 mar 25 ]. Disponível em: https://coronavirus.saude.gov.br/

5. Liang W, Guan W, Chen R, et al. Cancer patients in SARS-CoV-2 infection: a nationwide analysis in China. Lancet. 2020; 21(3):335-7. doi: https://doi.org/10.1016/ S1470-2045(20)30096-6

6. British Columbia Cancer [Internet]. Canada: BC Cancer Agency; c2020. COVID-19 and cancer treatments: information for patients; 2020 Apr 23 [cited 2020 May 26]. Available from: http://www.bccancer. bc.ca/about/news-stories/news/2020/covid-19-andcancer-treatments

7. Cancer Research Uk [Internet]. United Kingdom: Cancer Research Uk; [2002]. Coronavirus (COVID-19) and cancer; [reviewed 2020 Apr 30; cited 2020 May 26]. Available from: https://www.cancerresearchuk.org/aboutcancer/cancer-in-general/coronavirus-and-cancer

8. Sociedade Española de Oncología Médica [Internet]. Madrid: SEOM; [2020]. Revisiones científicas sobre COVID-19; 2020 Mar 17 [cited 26 Mar 2020]. Disponível em: https://seom.org/revisiones-cientificassobre-covid-19

9. Sociedade Brasileira de Oncologia Clínica [Internet]. São Paulo: SBOC; c2017. Especial SBOC coronavírus (covid-19); [2020] [acesso 2020 mar 26]. Disponível em: https://coronavirus.sboc.org.br/coronavirus/

10. Sidaway P. COVID-19 and cancer: what we know so far. Nat Rev Clin Oncol. 2020 Apr 7. doi: https://doi. org/10.1038/s41571-020-0366-2

11. Szklo M. Epidemiologia translacional: algumas considerações. Epidemiol Serv Saúde. 2015;24(1):16172. doi: https://doi.org/10.5123/S167949742015000100018

12. Yu J, Ouyang W, Chua MLK, et al. SARS-CoV-2 transmission in patients with cancer at a tertiary care hospital in Wuhan, China. JAMA Oncol. 2020 Mar 25:e200980. doi: https://doi.org/10.1001/ jamaoncol.2020.0980 Epub ahead of print. PubMed PMID: 32211820.

13. Desai A, Sachdeva S, Parekh T, et al. COVID-19 and cancer: lessons from a pooled meta-analysis. JCO Glob Oncol. 2020 Apr;6:557-9. doi: http://doi.org/10.1200/ GO.20.00097

14. Thuler LCS, Melo AC. Sars-CoV-2/Covid-19 em pacientes com câncer. Rev Bras Cancerol. 2020;66(2):e00970. doi: https://doi.org/10.32635/2176-9745. RBC.2020v66n2.970.

15. Wang B, Li R, Lu Z, et al. Does comorbidity increase the risk of patients with COVID-19: evidence from metaanalysis. Aging (Albany NY). 2020 Apr 8;12(7):6049-57. doi: https://doi.org/10.18632/aging. 103000

16. Emami A, Javanmardi F, Pirbonyeh N, et al. Prevalence of underlying diseases in hospitalized patients with covid-19: a systematic review and meta-analysis. Arch Acad Emerg Med [Internet]. 2020 [cited 2020 Mar 26];8(1):e35. Available from: https://www.ncbi.nlm.nih. gov/pmc/articles/PMC7096724/pdf/aaem-8-e35.pdf

17. Salako O, Okunade K, Allsop M, et al. Upheaval in cancer care during the COVID-19 outbreak. Ecancermedicalscience. 2020;14:ed97. doi: https://doi. org/10.3332/ecancer.2020.ed97

18. Zhang L, Zhu F, Xie L, et al. Clinical characteristics of COVID-19-infected cancer patients: a retrospective case study in three hospitals within Wuhan, China. Ann Oncol. 2020 Mar 26. https://doi.org/10.1016/j. annonc.2020.03.296 Epub ahead of print. PubMed PMID: 32224151.

19. Ma J, Yin J, Qian Y, et al. Clinical characteristics and prognosis in cancer patients with COVID-19: a single center's retrospective study [letter]. J Infect. 2020. doi: https://doi.org/10.1016/j.jinf.2020.04.006

20. Shi Y, Yu X, Zhao H, et al. Host susceptibility to severe COVID-19 and establishment of a host risk score: findings of 487 cases outside Wuhan. Crit Care. 2020;24(1):108. doi: https://doi.org/10.1186/s13054020-2833-7

21. Wang $\mathrm{D}, \mathrm{Hu} \mathrm{B}, \mathrm{Hu} \mathrm{C}$, et al. Clinical characteristics of 138 hospitalized patients with 2019 novel coronavirus- 
infected pneumonia in Wuhan, China. JAMA. 2020 Feb 7;323(11):1061-9. doi: https://doi.org/10.1001/ jama.2020.1585

22. Novel Coronavirus Pneumonia Emergency Response Epidemiology Team. [The epidemiological characteristics of an outbreak of 2019 novel coronavirus diseases (COVID-19) in China]. Zhonghua Liu Xing Bing Xue Za Zhi.2020;41(2):145-51. doi: https://doi. org/10.3760/cma.j.issn.0254-6450.2020.02.003 China

23. Guan WJ, Liang WH, Zhao Y, et al. Comorbidity and its impact on 1590 patients with Covid-19 in China: a nationwide analysis. Eur Respir J. 2020 Mar 26. doi: https://doi.org/10.1183/13993003.00547-2020 Epub ahead of print. PubMed PMID: 32217650.

24. Xia Y, Jin R, Zhao J, et al. Risk of COVID-19 for patients with cancer. Lancet Oncol. 2020;21(4):e180. doi: http:// doi.org/10.1016/S1470-2045(20)30150-9

25. Mourey L, Falandry C, de Decker L, et al. Taking care of older patients with cancer in the context of COVID-19 pandemic. Lancet Oncol. 2020;21(5):e236. doi: https:// doi.org/10.1016/S1470-2045(20)30229-1

26. Bouffet E, Challinor J, Sullivan M, et al. Early advice on managing children with cancer during the COVID-19 pandemic and a call for sharing experiences. Pediatr Blood Cancer. 2020 Apr 2:e28327. doi: https://doi. org/10.1002/pbc.28327 Epub ahead of print. PubMed PMID: 32239747.

27. Al-Quteimat OM, Amer AM. The impact of the COVID-19 pandemic on cancer patients. Am J Clin Oncol. 2020 April 16. doi: https://doi.org/10.1097/ COC.0000000000000712 Epub ahead of print. PubMed PMID: 32304435. 